\title{
Joint Multi-baseline SAR Interferometry
}

\author{
G. Fornaro \\ Istituto per il Rilevamento Elettromagnetico dell'Ambiente (IREA), Consiglio Nazionale delle Ricerche (CNR), \\ via Diocleziano 38, 80124 Napoli, Italy \\ Email:fornaro.g@irea.cnr.it
}

A. Monti Guarnieri

Dipartimento di Elettronica e Informazione, Politecnico di Milano, Piazza Leonardo da Vinci 32, 20133 Milano, Italy Email:monti@elet.polimi.it

\section{A. Pauciullo}

Istituto per il Rilevamento Elettromagnetico dell'Ambiente (IREA), Consiglio Nazionale delle Ricerche (CNR), via Diocleziano 38, 80124 Napoli, Italy

Email:pauciullo.a@irea.cnr.it

\section{S. Tebaldini}

Dipartimento di Elettronica e Informazione, Politecnico di Milano, Piazza Leonardo da Vinci 32, 20133 Milano, Italy Email: tebaldini@elet.polimi.it

Received 5 August 2004; Revised 27 December 2005

\begin{abstract}
We propose a technique to provide interferometry by combining multiple images of the same area. This technique differs from the multi-baseline approach in literature as (a) it exploits all the images simultaneously, (b) it performs a spectral shift preprocessing to remove most of the decorrelation, and (c) it exploits distributed targets. The technique is mainly intended for DEM generation at centimetric accuracy, as well as for differential interferometry. The problem is framed in the contest of single-input multiple-output (SIMO) channel estimation via the cross-relations (CR) technique and the resulting algorithm provides significant improvements with respect to conventional approaches based either on independent analysis of single interferograms or multi-baselines phase analysis of single pixels of current literature, for those targets that are correlated in all the images, like for long-term coherent areas, or for acquisitions taken with a short revisit time (as those gathered with future satellite constellations).
\end{abstract}

Keywords and phrases: synthetic aperture radar, interferometry, radar data processing, terrain mapping.

\section{INTRODUCTION}

The present and future availability of cooperative spaceborne, multipurpose SAR (synthetic aperture radar) sensors makes frequent coverage of the same scene possible. Both large ground coverage at coarse resolution and reduced ground coverage at fine resolution will require future SAR processing to deal with a large number of data sets, acquired from different viewing (looking) angles, of the same scene.

The potentialities intrinsic to cubes of data that will be available with future constellations have only been partially addressed in present and past literature. Most of the efforts have been addressed to the exploitation of permanent

This is an open access article distributed under the Creative Commons Attribution License, which permits unrestricted use, distribution, and reproduction in any medium, provided the original work is properly cited. scatterers (PS) [1], that is, those structures that are stable over the long term, the main interest being in monitoring changes and subsidence. Unlike the PS approach, we consider those distributed scatterers that maintain a good degree of correlation throughout the set of images. The applications foreseen for these techniques are mainly DEM generation at centimetric accuracy or maybe short-term monitoring by differential interferometry. The object of this paper is to establish a theoretical framework that can extend the concept of optimal spectral shift filtering (SSF) $[2,3,4,5]$ to the case when more than two images are available. The idea is to exploit all the images jointly, like in the ML approach, proposed in [6]. However, much better results can be expected due to the use of SSF prefiltering. In fact, this technique merges into the final multi-baseline interferogram the correlated information, the incoherent information (coming from geometric decorrelation) having been filtered out. Obviously, other 


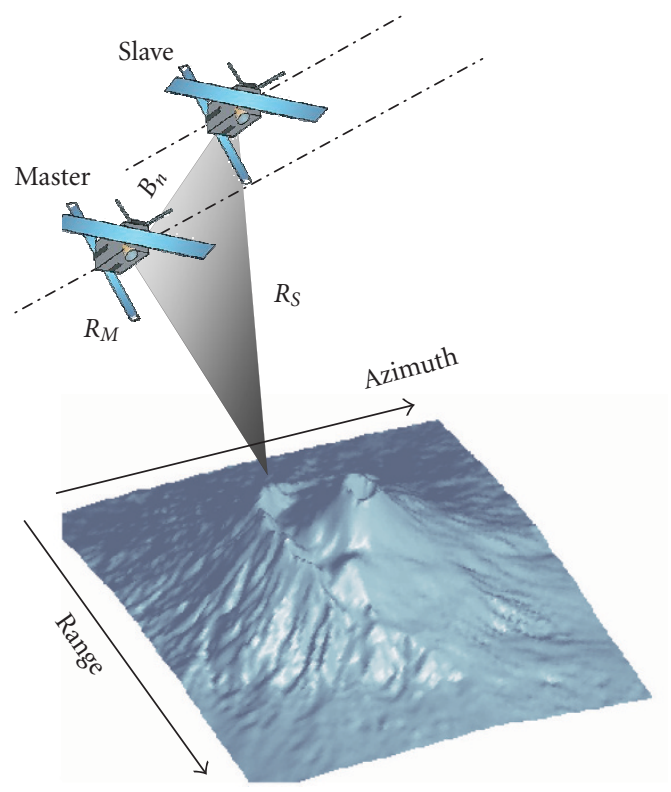

(a)

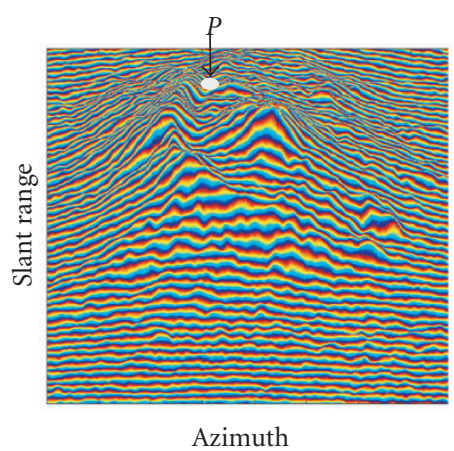

(b)

FIGURE 1: Interferometric SAR geometry: a master and a slave sensor, at the same azimuth position, are shown. The travel path distance, converted into phases, is shown in (b).

decorrelation sources (thermal, temporal, etc.) are not affected by the proposed algorithm.

We emphasize the fact that the proposed technique is aimed at establishing a framework wherein several acquisitions under different modes (STRIPMAP, SCANSAR, etc.) $[7,8]$ can be optimally combined to exploit to the maximum information on the underlying topography. Also combinations with different carrier frequencies, like ERS-2 and ENVISAT, may be considered.

The paper is organized as follows. Section 2 introduces multichannel interferometry and recasts the topography estimation as a SIMO blind estimation problem. Section 3 addresses the use of CR, typically adopted in the contest of SIMO problems. In Section 4 we discuss the relevant case of single-pass (two-channel) interferometry and show that it can be viewed as a particular case of our more general multichannel approach. Section 5 addresses implementation issues; for easy implementation and to overcome problems related to atmospheric phase aberration in real data, the estimation is casted in terrain slopes. Nevertheless easy slope integrations can be carried out, for instance, via standard least square (LS) approaches [9]. Section 6 shows some results on simulated data to validate the theory.

\section{PROBLEM FORMULATION}

Let us consider the multi baseline geometry in Figure 1: this geometry is fairly conventional, and the reader is referred to for example $[7,8,10]$ for a general view of SAR interferometry. The interferogram is the Hermitian product of the two images: $i=y_{0} \cdot y_{i}^{*}$, coregistered in the slant range, azimuth reference of the master acquisition. Its phase, shown in Figure $1 \mathrm{~b}$ is proportional to the travel phase difference between the two acquisitions

$$
\Delta \varphi=\frac{4 \pi}{\lambda}\left(R_{i}(P)-R_{0}(P)\right),
$$

where $R_{0}(P)$ and $R_{i}(P)$ are respectively the slant range of the master and slave antennas to the target point $P$, and $\lambda$ is the transmitted wavelength. A constant sloped terrain contributes to the interferogram as a linear phase:

$$
\phi=-\omega_{0} \frac{B_{n i}}{r_{0} \tan (\theta-\alpha)} t
$$

$t$ being slant range fast time, $B_{n i}$ the normal baseline, $r_{0}$ the closest approach, $\omega_{0} / 2 \pi$ the carrier frequency, $\theta$ the local incidence angle, and $\alpha$ the terrain slope. Note that the phase scales linearly with the baseline.

For the sake of simplicity and without loosing generality, let us assume a $1 \mathrm{D}$ model, where $P$ varies in the slant range direction $P=P(t)$, in order to approach the problem of baseline decorrelation that mostly affects range (see [11]). Furthermore, the usual fine sampling in azimuth direction is exploited to perform a preliminary complex multilook (average), to get a reasonable SNR in each acquisition. To account for channel differences in the azimuth direction (Doppler centroid variation, different operative modes, etc.), the theory can be extended to the 2D case, along the lines addressed in the following.

The problem is to estimate the constant terrain slope $\alpha$ in (2) starting from the availability of several interferometer 
pairs. Its formulation is an extension of the optimal MMSE (minimum mean square error) estimate given in [5] for the case of multiple $(\geq 2)$ acquisitions.

\subsection{Forward model}

Let us assume $N$ acquisitions, including one master and $(N-1)$ slave images, and $M$ samples (range bins) out of each acquisition. According to (1), and to the formulation in [5], we can express each single acquisition, coregistered in the reference frame of the master, as a filtered version of the (large bandwidth) reflectivity $\gamma$ :

$y_{i}(P)=f_{i}(P) *\left[\exp \left(j \frac{4 \pi}{\lambda}\left(R_{i}(P)-R_{0}(P)\right)\right) \gamma(P)\right]+w_{i}(P)$,

where $*$ is the convolution product symbol, $P$ is a target on the ground, $y_{i}$ the focused signal of the $i$ th acquisition, $R_{i}-R_{0}$ the travel path difference between the master (without loss of generality indexed by " 0 ") and the slave (indexed by " $i$," $i=0, \ldots, N-1$ ), and $f_{i}$ the post-focusing SAR impulse response function. The term $w_{i}$ is an additive noise contribution that accounts for all the decorrelation sources like thermal noise, volume scattering, and temporal decorrelation $[7,8]$ (but not the baseline decorrelation, which is the one we are trying to remove): we assume this noise white within the system bandwidth.

We will make the assumption of homogenous indefinite scatterer, so that $\gamma(P)$ can be modeled as a realization of a complex circular Normal process, uncorrelated in both time and spectral domains; note, however, that all the images are fed by the same realization of this process. We convert the model in (3) to a discrete one and, to avoid alias, we assume a sampling rate compatible with the bandwidth of all the acquisitions and the frequency shifts. As an example, for a typical set of ERS-ENVISAT acquisitions, the baseline dispersion demands for an oversampling of a factor 4 (see discussions in [5]). The model (3) leads to the following matrix formulation:

$$
\mathbf{y}_{i}=\mathbf{F}_{i} \Phi_{i} \gamma+\mathbf{w}_{i}=\mathbf{H}_{i} \boldsymbol{\gamma}+\mathbf{w}_{i}, \quad i=0, \ldots, N-1,
$$

where the matrixes and vectors are shown in bold notation. In particular, we assume that the impulse response on each channel extends for $L$ samples; then we require $D=M+L-1$ samples of the source $\gamma(P)$. The vectors and matrixes involved in (4) are

(i) $\mathbf{y}_{i}$ is the column vector $[M, 1]$ that corresponds to the complex SAR image, the data, coregistered in the reference of the master:

$$
\mathbf{y}_{i}=\left[\begin{array}{llll}
y_{0} & \cdots & \cdots & y_{M-1}
\end{array}\right]^{T},
$$

where the superscript $T$ stands for matrix transposition, and we assume row and column indices starting from 0 ;

(ii) $y$ is the column vector $[D, 1]$ that represents the source reflectivity; (iii) $\boldsymbol{\Phi}_{i}$ is a diagonal modulation matrix $[D, D]$ that expresses the topographic-dependent contributions:

$$
\boldsymbol{\Phi}_{i}=\left[\begin{array}{cccc}
\phi_{i}(0) & 0 & \cdots & 0 \\
0 & \phi_{i}(1) & \cdots & 0 \\
\vdots & \vdots & \vdots & \vdots \\
0 & 0 & \cdots & \phi_{i}(D-1)
\end{array}\right]
$$

its element on the diagonal being

$$
\phi_{i}(k)=\exp \left(j \frac{4 \pi}{\lambda}\left(R_{i}\left(P_{k}\right)-R_{0}\left(P_{k}\right)\right)\right) ;
$$

(iv) $\mathbf{F}_{i}$ is the filter matrix $[M, D]$ that is Toeplitz and contains the impulse response of the equivalent SAR endto-end channel (well approximated by an ideal bandpass),

$$
\mathbf{F}_{i}=\left[\begin{array}{cccccc}
f_{i, L-1} & f_{i, L-2} & \cdots & f_{i, 0} & 0 & 0 \\
0 & f_{i, L-1} & \cdots & \cdots & f_{i, 0} & 0 \\
\vdots & \vdots & \vdots & \vdots & \vdots & \vdots \\
0 & 0 & f_{i, L-1} & \ldots & \ldots & f_{i, 0}
\end{array}\right]
$$

(v) $\mathbf{H}_{i}$ is a matrix $[M, D]$,

$$
\mathbf{H}_{i}=\mathbf{F}_{i} \boldsymbol{\Phi}_{i},
$$

that represents the channel dependent on the slope $\alpha$ to be estimated. Note that the channel is now linear, but space-variant due to the modulation matrix $\boldsymbol{\Phi}_{i}$, hence $\mathbf{H}_{i}$ is not block Toeplitz as usually assumed in literature;

(vi) $\mathbf{w}_{i}$ is the additive noise contribution; it has the same size as $\mathbf{y}_{i}$.

The model (4) lies within the SIMO blind estimation, where a common unknown parameter of the channels $\mathbf{H}_{i}$ is to be retrieved from the outputs, $\mathbf{y}_{i}$, only some information on the inputs being available. In this paper, we will assume $\gamma$ coming from a homogenous, white target. The problem of channel estimation will not explicitly require the estimate of these sources.

The problem is patently unsolvable if only one channel is given, whereas solutions can be formulated for $N \geq 2$ channels, as for the case of SAR interferometry. Indeed there is widespread literature on the topic, as similar models are found in many fields: estimate of direction of arrivals (DOA), wireless cellular networks, tomography, and so forth: the reader is referred to paper [12] for a summary on blind deconvolution techniques for SIMO problems.

Let us assume the SIMO model in Figure 2; each SAR acquisition is represented by a different channel as in (4). This same model is usually represented in the single block-matrix format:

$$
\mathbf{y}=\mathbf{H} \boldsymbol{\gamma}+\mathbf{w},
$$




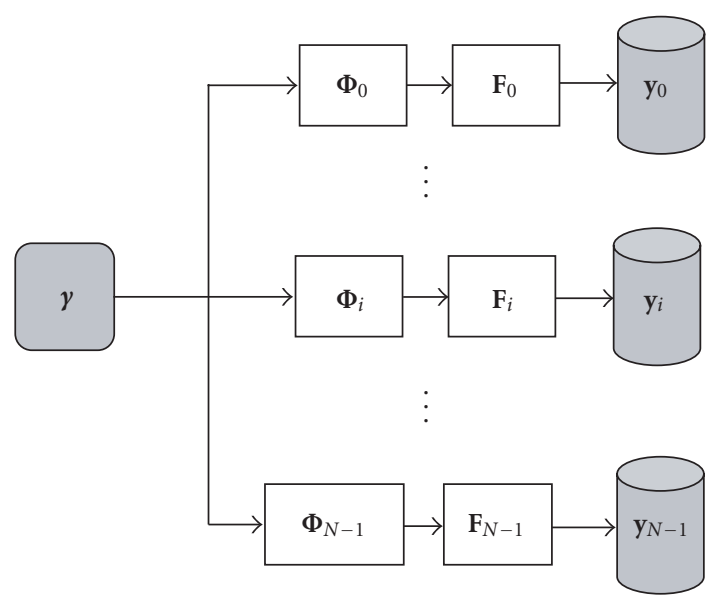

FIGURE 2: Multi-baseline interferometric SAR system, modeled as a SIMO system corresponding to (4).

where the input and output vectors are obtained by stacking all the $N$ inputs and outputs of each channel:

$$
\begin{aligned}
\mathbf{y} & =\left[\begin{array}{lllll}
\mathbf{y}_{0}^{T} & \cdots & \mathbf{y}_{i}^{T} & \cdots & \mathbf{y}_{N-1}^{T}
\end{array}\right]^{T}, \\
\mathbf{w} & =\left[\begin{array}{lllll}
\mathbf{w}_{0}^{T} & \cdots & \mathbf{w}_{i}^{T} & \cdots & \mathbf{w}_{N-1}^{T}
\end{array}\right]^{T},
\end{aligned}
$$

$\mathbf{y}$ and $\mathbf{w}$ being then column vectors of size $[N M, 1]$. The channel matrix $\mathbf{H}$ in (10) is also a block matrix of size $[N M, D]$ made by stacking all the channels $\mathbf{H}_{i}$ one upon another:

$$
\begin{aligned}
\mathbf{H} & =\left[\begin{array}{ccccc}
\mathbf{H}_{0}^{T} & \cdots & \mathbf{H}_{i}^{T} & \cdots & \mathbf{H}_{N-1}^{T}
\end{array}\right]^{T} \\
= & {\left[\begin{array}{cccc}
\phi_{0}(0) f_{L-1} & \cdots & \cdots & 0 \\
0 & \cdots & \cdots & 0 \\
0 & \phi_{0}(D-L) f_{L-1} & \cdots & \phi_{0}(D-1) f_{0} \\
& \cdots & & \\
& \mathbf{H}_{i} & & \\
& \cdots & &
\end{array}\right], }
\end{aligned}
$$

where the dependence of the bandpass filter on index " $i$ " is neglected for the sake of simplicity.

\section{SIMO ESTIMATE BY CROSS-RELATION}

The CR approach provides the optimal estimate of the parameters describing the channels (in our case the terrain slope) for a large SNR, and closely follows the methodology proposed by the seminal paper of Xu et al. [13] and many others in almost the same period (see [12]). For a generic case of SIMO space invariant channel, the CR approach exploits the commutative property of the convolution to write the following equation:

$$
\begin{gathered}
y_{i}(n) * h_{j}(n)=y_{j}(n) * h_{i}(n), \\
y_{i}(n) * h_{j}(n)-y_{j}(n) * h_{i}(n)=0,
\end{gathered}
$$

where $y_{i}$ is the sequence at the output of the channel " $i$," and $h_{j}$ is the impulse response of the channel " $j$," linear and space

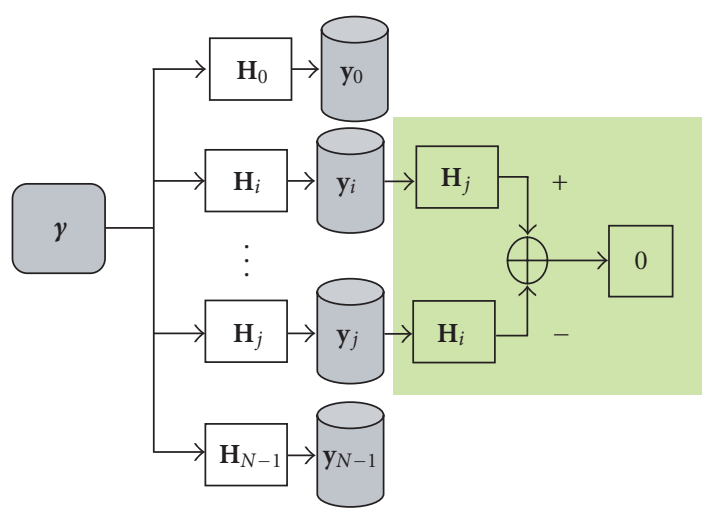

FIGURE 3: Cross-relations: the signal blocking transformation shown here is applied to all the channel pairs to span the null space of the channel matrix.

invariant, and $n$ is the pixel. Note that (14) acts as a sort of "signal blocking transformation": the signal is cancelled, hence the residual should be zero, or better still should attain minimal energy in the presence of noise. The signal blocking transformation is exemplified in Figure 3 for the case of a general multichannel system. The way $\mathrm{CR}$ achieves the estimate of all the channels is just by extending (13) to all the two channels combinations and then solving the resulting system equations, either in exact form, or as LS (least square) solution.

These transformations cannot be directly extended to the interferometric SAR model formulated in Section 2.1 as the model is nonstationary due to the modulation matrixes $\boldsymbol{\Phi}_{i}(\alpha)$ ( $\alpha$ being the parameter to be estimated). However, in the noiseless case, the modulation of each channel introduced by the topographic-dependent term can be reversed, leading to the intermediate vector:

$$
\mathbf{y}_{i}^{\prime}=\boldsymbol{\Phi}_{i}^{*}(x) \mathbf{y}_{i}=\boldsymbol{\Phi}_{i}^{*}(x) \mathbf{F}_{i} \boldsymbol{\Phi}_{i}(\alpha) \boldsymbol{\gamma},
$$

where $x$ is the unknown parameter and the suffix $*$ stands for matrix transposition and conjugation. Now let us define the vector

$$
\begin{aligned}
\mathbf{J}_{i j}(x) & =\boldsymbol{\Phi}_{j}^{*}(x) \mathbf{F}_{j} \boldsymbol{\Phi}_{j}(x) \mathbf{y}_{i}^{\prime}-\boldsymbol{\Phi}_{i}^{*}(x) \mathbf{F}_{i} \boldsymbol{\Phi}_{i}(x) \mathbf{y}_{j}^{\prime} \\
& =\left[\boldsymbol{\Phi}_{j}^{*}(x) \mathbf{F}_{j} \boldsymbol{\Phi}_{j i}(x)-\boldsymbol{\Phi}_{i}^{*}(x) \mathbf{F}_{i} \boldsymbol{\Phi}_{i j}(x)\right]\left[\begin{array}{c}
\mathbf{y}_{i} \\
\mathbf{y}_{j}
\end{array}\right],
\end{aligned}
$$

where the definition

$$
\boldsymbol{\Phi}_{i j}(x) \stackrel{\circ}{=} \boldsymbol{\Phi}_{i}(x) \Phi_{j}^{*}(x),
$$

a diagonal matrix with the overall phase between two channels, is introduced. Note that the vectors $\mathbf{y}_{i}$ should be zeropadded to an extent $[1, D]$ to make (16) meaningful. Based on the CR theory, it can be easily shown that, when the modulation matrixes have a linear phase variation (like for constant sloped terrain, i.e, the case we are interested in) the vector (16) is minimized (in the LS sense) for $x=\alpha$. 
A comment on the approximations implied in the presence of changing slopes, like for rolling topography, is included at the end of Section 4.2 .

The idea in the paper [13] was to solve all the relations of type $\mathbf{J}_{i j}(x)=\mathbf{0}$ simultaneously for all the channel pairs; this leads to the equation system

$$
\mathscr{D}\left[\mathbf{H}^{\prime}(x)\right] \mathbf{y}^{\prime}=\mathbf{0},
$$

where the transformation $\mathscr{D}(\cdot)$ and the channel $\mathbf{H}^{\prime}(x)$ are defined as ( 3 channels have been assumed)

$$
\begin{gathered}
\mathbf{H}_{i}^{\prime}(x)=\boldsymbol{\Phi}_{i}^{*}(x) \mathbf{F}_{i} \boldsymbol{\Phi}_{i}(x), \\
\mathbf{H}^{\prime}(x)=\left[\begin{array}{lll}
\mathbf{H}_{0}^{\prime}(x) & \mathbf{H}_{1}^{\prime}(x) & \mathbf{H}_{2}^{\prime}(x)
\end{array}\right], \\
\mathcal{D}\left[\mathbf{H}^{\prime}(x)\right]=\left[\begin{array}{ccc}
\mathbf{H}_{1}^{\prime}(x) & -\mathbf{H}_{0}^{\prime}(x) & \mathbf{0}(x) \\
\mathbf{H}_{2}^{\prime}(x) & \mathbf{0}(x) & -\mathbf{H}_{0}^{\prime}(x) \\
\mathbf{0} & \mathbf{H}_{2}^{\prime}(x) & -\mathbf{H}_{1}^{\prime}(x)
\end{array}\right] .
\end{gathered}
$$

Note that the number of equations is

$$
P=\left(\begin{array}{c}
N \\
2
\end{array}\right)=\frac{N !}{2 !(N-2) !},
$$

hence $\mathscr{D}\left[\mathbf{H}^{\prime}(x)\right]$ will be a tall matrix $[P D, N D]$.

In $[13]$, it is shown that $\mathscr{D}\left[\mathbf{H}^{\prime}(x)\right]$ is close to full rank, having only one singular value that corresponds to an unknown constant scaling applied to all the channels, provided that (a) the channels are coprime or, (b) they do not share common zeros. In the interferometric SAR case, where channels are strongly upsampled and different spectral shifts [2] can be experienced, there may be many common zeros, and it may even happen that the set of (14) is undermined. We then search for the LS solution for (18), that is, a terrain slope $\hat{\alpha}$ that minimizes the $L_{2}$ norm of the vector $\mathcal{D}\left[\mathbf{H}^{\prime}(x)\right] \mathbf{y}^{\prime}$. For this purpose let us introduce the definition

$$
\begin{aligned}
& \mathbf{R}=\mathscr{D}\left(\mathbf{H}^{\prime}\right)^{*} \mathcal{D}\left(\mathbf{H}^{\prime}\right) \\
& =\left[\begin{array}{ccc}
\mathbf{H}_{1}^{\prime *} & \mathbf{H}_{2}^{\prime *} & 0 \\
-\mathbf{H}_{0}^{\prime *} & 0 & \mathbf{H}_{2}^{\prime *} \\
0 & -\mathbf{H}_{0}^{\prime *} & -\mathbf{H}_{1}^{\prime *}
\end{array}\right]\left[\begin{array}{ccc}
\mathbf{H}_{1}^{\prime} & -\mathbf{H}_{0}^{\prime} & 0 \\
\mathbf{H}_{2}^{\prime} & 0 & -\mathbf{H}_{0}^{\prime} \\
0 & \mathbf{H}_{2}^{\prime} & -\mathbf{H}_{1}^{\prime}
\end{array}\right]
\end{aligned}
$$

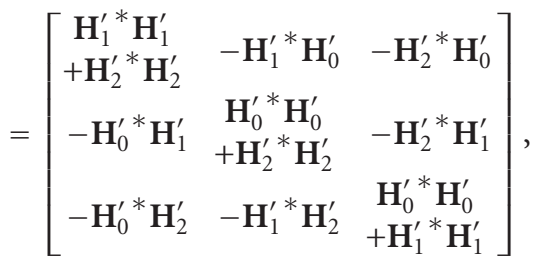

where the dependence of the channels on the unknown $x$ is neglected for the sake of simplicity. In a general case we obtain the expression

$$
\begin{gathered}
\mathbf{R}=\left[\mathbf{R}_{i, j}\right]_{i, j=0}^{N-1}, \\
\mathbf{R}_{i, j}= \begin{cases}\sum_{k=0 ; k \neq i}^{N-1} \mathbf{H}_{k}^{\prime *} \mathbf{H}_{k}^{\prime}, & i=j, \\
-\mathbf{H}_{j}^{\prime *} \mathbf{H}_{i}^{\prime}, & i \neq j .\end{cases}
\end{gathered}
$$

The LS solution leads to the minimization

$$
\begin{aligned}
\hat{\alpha} & =\underset{x}{\operatorname{argmin}}\left(\mathbf{y}^{\prime *} \mathbf{R} \mathbf{y}^{\prime}\right) \\
& =\underset{x}{\operatorname{argmin}}\left(\sum_{i=0}^{N-1} \sum_{j=0}^{N-1} \mathbf{y}_{i}^{\prime *} \mathbf{R}_{i, j} \mathbf{y}_{j}^{\prime}\right) \\
& =\underset{x}{\operatorname{argmax}}\left(\begin{array}{c}
\sum_{j=0}^{N-1} \sum_{i=0 ; i \neq j}^{N-1} \mathbf{y}_{j}^{\prime *} \mathbf{H}_{i}^{\prime *} \mathbf{H}_{j}^{\prime} \mathbf{y}_{i}^{\prime} \\
-\sum_{j=0}^{N-1} \sum_{i=0 ; i \neq j}^{N-1} \mathbf{y}_{j}^{\prime *} \mathbf{H}_{i}^{\prime *} \mathbf{H}_{i}^{\prime} \mathbf{y}_{j}^{\prime}
\end{array}\right) .
\end{aligned}
$$

We end up, as for any $L_{2}$ norm problem, in minimizing the difference between the energy from all the channels after filtering (cochannel energy):

$$
\sum_{j=0}^{N-1} \sum_{i=0 ; i \neq j}^{N-1}\left(\mathbf{H}_{i}^{\prime} \mathbf{y}_{j}^{\prime}\right)^{*}\left(\mathbf{H}_{i}^{\prime} \mathbf{y}_{j}^{\prime}\right)
$$

and the energy from the cross-channels:

$$
\sum_{j=0}^{N-1} \sum_{i=0 ; i \neq j}^{N-1}\left(\mathbf{H}_{i}^{\prime} \mathbf{y}_{j}^{\prime}\right)^{*}\left(\mathbf{H}_{j}^{\prime} \mathbf{y}_{i}^{\prime}\right)
$$

The cochannel terms in (24) are useless for estimating the channel phase (a common knowledge in SAR interferometry, where the single channel is never used alone), as the sources are normal distributed. Therefore the contributions in (24) are dropped.

The terms for multichannel interferometry are then the cross-channel ones, that is, those involving cross-channel data correlations in (25). The LS solution therefore leads to the following maximization:

$$
\begin{aligned}
\widehat{\alpha} & =\underset{x}{\operatorname{argmax}}\left(\sum_{j=0}^{N-1} \sum_{i=0 ; i \neq j}^{N-1} \mathbf{y}_{j}^{\prime *} \mathbf{H}_{i}^{\prime *} \mathbf{H}_{j}^{\prime} \mathbf{y}_{i}^{\prime}\right) \\
& =\underset{x}{\operatorname{argmax}}\left(\sum_{j=0}^{N-1} \sum_{i=j+1}^{N-1} 2 \operatorname{Re}\left(\mathbf{y}_{j}^{\prime *} \mathbf{H}_{i}^{\prime *} \mathbf{H}_{j}^{\prime} \mathbf{y}_{i}^{\prime}\right)\right),
\end{aligned}
$$

that is the summation of terms like

$$
\mathbf{y}_{j}^{\prime *} \mathbf{H}_{i}^{\prime *} \mathbf{H}_{j}^{\prime} \mathbf{y}_{i}^{\prime}=\mathbf{y}_{j}^{*} \boldsymbol{\Phi}_{j i}(x) \mathbf{F}_{i}^{*} \boldsymbol{\Phi}_{i j}(x) \mathbf{F}_{j} \boldsymbol{\Phi}_{j i}(x) \mathbf{y}_{i}
$$

\section{SINGLE-PASS SAR INTERFEROMETRY}

Let us approach the conventional interferometric system as a special case of multipass interferometry with only $N=2$ channels, that is, the minimum number of channels to ensure a solution in the case of unknown input.

Figure 4 shows, for the two-channel system, the forward model (shaded in gray) as well as the linear estimate of both 


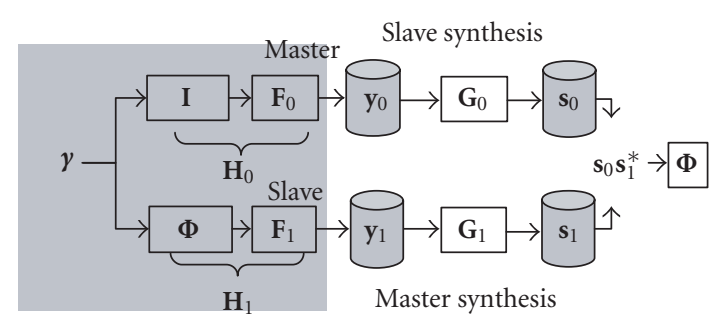

FIgURE 4: Forward model and linear estimate for 2-channel conventional SAR interferometry.

master and slave acquisition needed to filter out noise contribution associated with spatial decorrelation phenomena. This estimate is given by the two vectors:

$$
\begin{aligned}
& \mathbf{s}_{0}=\mathbf{G}_{0} \mathbf{y}_{0}, \\
& \mathbf{s}_{1}=\mathbf{G}_{1} \mathbf{y}_{1},
\end{aligned}
$$

computed as linear, space variant filtering of the master and slave images respectively, where

$$
\begin{gathered}
\mathbf{G}_{0}=\mathbf{F}_{1} \boldsymbol{\Phi}, \\
\mathbf{G}_{1}=\mathbf{F}_{0} \boldsymbol{\Phi}^{*} .
\end{gathered}
$$

Obviously a preliminary DEM estimate should be available. The two signals correspond to the synthesis of the master reflectivity from the slave, and the slave reflectivity of the master, derived as an approximation of the optimal MMSE approach in paper [5]. These estimates default to the optimal spectral shift filtering for the case of constant slope [2], also known as common band (CB) filter. Following the filtering in (28), the useful interferogram is estimated as

$$
\widehat{\boldsymbol{\Phi}}=\arg \left(\mathbf{s}_{0} \circ \mathbf{s}_{1}^{*}\right)=\arg \mathbf{I}_{10},
$$

where $\widehat{\Phi}$ is the vector collecting the phases to be estimated, 。 the element-by-element vector (matrix) product, and $\mathbf{I}_{10}$ the prefiltered, complex interferogram.

We now demonstrate that the above interferometric processing can be considered a particular case of the more general multichannel processing addressed in the previous section. For this purpose, let us assume $N=2$ and relax the hypothesis of constant slope topography (see Section 4.2 on the validity of such assumption). In this case our unknown is the whole interferometric phase matrix $\boldsymbol{\Phi}$ and, according to (26) and (27), we have

$$
\begin{aligned}
\widehat{\boldsymbol{\Phi}} & =\underset{\Psi}{\operatorname{argmax}}\left[\operatorname{Re}\left(\mathbf{s}_{1}^{*} \boldsymbol{\Psi}^{*} \mathbf{s}_{0}\right)\right] \\
& =\underset{\psi_{0}, \ldots, \psi_{D-1}}{\operatorname{argmax}}\left(\operatorname{Re}\left(\sum_{k=0}^{D-1} \exp \left(-j \psi_{k}\right) s_{1, k}^{*} s_{0, k}\right)\right),
\end{aligned}
$$

where $\Psi=\operatorname{diag}\left(\exp \left(-j \psi_{0}\right), \ldots, \exp \left(-j \psi_{D-1}\right)\right)$ is the unknown $D \times D$ diagonal matrix and $s_{i, k}$ is the $i$ th element of vectors $\mathbf{s}_{i}, i=0,1$, exactly defined as in (28). As for [5], the solution of (31) is obtained via (30).

In the case of constant slope, we should substitute (29) by the filter

$$
\begin{gathered}
\mathbf{G}_{0}=\mathbf{F}_{1} \boldsymbol{\Phi}(\bar{\alpha}), \\
\mathbf{G}_{1}=\mathbf{F}_{0} \boldsymbol{\Phi}^{*}(\bar{\alpha}),
\end{gathered}
$$

where $\bar{\alpha}$ is a starting constant slope value, possibly derived from an initial DEM of the area of interest. Obviously in this case the solution benefits from averaging $M$ samples along range:

$$
\hat{\alpha}=\underset{x}{\operatorname{argmax}}\left(\operatorname{Re}\left(\sum_{k=0}^{D-1} \exp (-j 2 \pi x k) s_{1, k}^{*} s_{0, k}\right)\right),
$$

where $\hat{\alpha}$ is obtained by $x$ by inverting the following expression (see (2)):

$$
x=\frac{f_{0}}{f_{s}} \frac{B_{n i}}{r_{0} \tan (\theta-\alpha)},
$$

$f_{s}$ being the sampling frequency.

In conclusion the proposed algorithm represents an extension of both classic interferometry and spectral shift filtering in a multipass system.

\subsection{The noisy case}

No considerations were given to the noisy case in CR, and the authors recognize this limit. However, in our case, we can exploit the MMSE approach in [5] to account for noise as an added weight in the MMSE estimates (28). This weight, as comes out from [5], is equal to $\left(1+\mathrm{SNR}^{-1}\right)^{-1}$, as it is usual for Wiener problems. Note that the weight applied to the crosscorrelation $\mathbf{s}_{1}^{*} \boldsymbol{\Psi}^{*} \mathbf{s}_{0}$ in (31) becomes

$$
g=\frac{1}{1+\mathrm{SNR}_{1}^{-1}} \frac{1}{1+\mathrm{SNR}_{2}^{-1}}
$$

that corresponds to the absolute value of the coherence. Furthermore, the weights should cut out from the combination (28) those baselines that are completely decorrelated, for example, at least when $|x|>1$ in (34), but in practice we need a greater margin, in order to provide enough independent samples for the coherence estimate. We can assume, for example, $g=0$ for $|x|>0.7$.

\subsection{Continuous-time domain interpretation}

Let us approach the continuous time-domain interpretation of the cross-relations in the two-channel case. The forward model (4) translates in time/frequency as follows:

$$
\begin{gathered}
y_{i}(t)=\left\{\gamma(t) \exp \left(-j \phi_{i}(t)\right)\right\} * f_{i}(t) \\
Y_{i}(f)=\left\{\Gamma(f) * \Phi_{i}(f)\right\} F_{i}(f)
\end{gathered} \quad \text { for } i=0,1,
$$


where capital letters denote the fourier transform and “ $*$ " stands for convolution. The cross-relations (16) are now expressed as follows:

$$
\begin{gathered}
\left(\left(y_{0}(t) \exp (-j \hat{\phi}(t))\right) * f_{1}(t)\right) \exp (-j \hat{\phi}(t)) \\
\simeq\left(y_{1}(t) \exp (j \hat{\phi}(t))\right) * f_{0}(t),
\end{gathered}
$$

where the equality holds in the $L_{2}$ norm. We further express (37) in the frequency domain, by exploiting (36):

$$
\left[\left(\Gamma(f) F_{0}(f) * \widehat{\Phi}(f)\right) F_{1}(f)\right] * \widehat{\Phi}^{*}(-f) .
$$

If we assume constant slope, $\Phi(f)=\delta\left(f+\Delta_{f}\right)=\widehat{\Phi}(f)$ (e.g., in correspondence of the optimum), the following relation holds:

$$
\left[\left(\Gamma \cdot F_{0} * \widehat{\Phi}\right) \cdot F_{1}\right] * \widehat{\Phi}^{*}(-f) \equiv \Gamma \cdot F_{0} \cdot F_{1}\left(f-\Delta_{f}\right),
$$

where we dropped the obvious frequency dependence of the terms. We notice that the nonstationary sequence of operators: modulation, filtering, and demodulation implied in the left-hand term of (39), becomes on the right-hand term a simple and stationary filtering. This filter is just the cascade of the master SAR channel and the modulated slave SAR channel. This property not only justifies the CR derivation (16), but also validates CR for all the cases in which (39) holds, at least as an approximation. We need the Fourier transform of the synthetic fringes to be close to impulsive, $\Phi(f) \simeq \delta\left(f+\Delta_{f}\right)$, henceforth we require the bandwidth of $\Phi(f)$ to be smaller than the inverse of the temporal support of the scene. In practice we accept "quasistationary slopes" that change smoothly in the estimation window, a fact already assessed analytically in the appendix of [4].

\section{IMPLEMENTATION: SLOPE ESTIMATE}

The MB channel estimate derived from CR has, in accordance with (23) and (26), been applied to a constant terrain slope. In the implementation we simplified the retrieving phase problem, just by estimating the pixel-to-pixel phase difference (PD) instead of the absolute phase value. This means that the maximization in (23) is carried out with respect to the phase variations $(\alpha)$ for a chosen reference baseline.

Following the approach suggested in (26), (31), and [5], we first performed the filtering (defined as common band in [5]), and then estimated the local slope, as phase difference. Moreover, the CB filtering was carried out with respect to an apriori reference PD pattern $(\bar{\alpha})$ that corresponded to our starting point for PD retrieval. Note that, to improve the slope estimation, such a reference phase is also used for the demodulation of the cross-channel interference (zero baseline steering). This demodulation, although not strictly necessary, allows us to improve the performance of the PD estimation process as for any phase retrieval algorithm. Let us start from (26) which we rewrite explicitly as

$$
\hat{\alpha}=\underset{x}{\operatorname{argmax}}\left(\sum_{j=0}^{N-1} \sum_{i=0 ; i \neq j}^{N-1} \mathbf{y}_{j}^{*} \boldsymbol{\Phi}_{j i}(\bar{\alpha}) \mathbf{F}_{i}^{*} \boldsymbol{\Phi}_{j i}(\bar{x}) \mathbf{F}_{j} \boldsymbol{\Phi}_{j i}(\bar{\alpha}) \mathbf{y}_{i}\right) .
$$

Letting $r i\{\cdot\}$ be the operator that evaluates the interference between adjacent pixels along the range, we introduce the following two signals:

$$
\begin{gathered}
\mathbf{y l}_{i j}(\bar{\alpha})=r i\left\{\boldsymbol{\Phi}_{i j}^{*}(\bar{\alpha}) \mathbf{F}_{i} \boldsymbol{\Phi}_{i j}(\bar{\alpha}) \mathbf{y}_{j}\right\}, \\
\mathbf{y r}_{i j}(\bar{\alpha})=r i\left\{\mathbf{F}_{j} \boldsymbol{\Phi}_{j i}(\bar{\alpha}) \mathbf{y}_{i}\right\},
\end{gathered}
$$

where, according to the position in (40), the suffixes 1 and $r$ stand, respectively, for left and right. Equations (41) and (42) carry out the spectral shift filtering; the additional modulation matrix $\boldsymbol{\Phi}_{i j}^{*}(\bar{\alpha})$ in (41) centers the signal interference on the known PD. The resulting maximization formula is written as

$$
\begin{gathered}
\hat{\alpha}=\bar{\alpha}+\hat{e}, \\
\hat{e}=\underset{e}{\operatorname{argmax}}\left[\sum_{j=0}^{N-1} \sum_{i=0 ; i \neq j}^{N-1} \mathrm{yl}_{\mathrm{ij}}^{*}(\overline{\mathrm{ff}}) \Phi_{\mathrm{ji}}(\mathrm{e}) \mathrm{yr}_{\mathrm{ij}}(\overline{\mathrm{ff}})\right] .
\end{gathered}
$$

In (43) we have explicitly highlighted the reference PD $(\bar{\alpha})$ and the wanted (unknown) PD (e) that, to distinguish from the $(\hat{\alpha})$, will be referred to as "excess PD."

Numerical implementation of (43) is now quite simple. For each range line of the image ( $M$ bins) and for each image pair, we build two $3 \mathrm{D}$ matrixes $(N \times N \times M-1) \mathrm{Y} L$ and $\mathrm{Y} R$. For each fixed range bin, the resulting $2 \mathrm{D}$ matrixes are Hermitian matrixes that correspond to the signals in (41) and (42), respectively. Element by element multiplication of $\mathrm{Y} L$ and $\mathrm{Y} R$ again leads, for each range pixel, to a Hermitian Matrix that describes the PD excess (with respect to the reference PD) at all the interferometric pairs present in the data. We build a 3D phase matrix

$$
\Phi 3=\mathbf{1}_{M-1} \otimes\left(\mathbf{f f}^{*}\right),
$$

with

$$
\mathbf{f}^{T}=\exp \left[-j \mathbf{a}^{T} e\right]
$$

where $\mathbf{a}$ is the baseline vector normalized to the reference baseline and e is the current testing PD excess, $\mathbf{1}_{M-1}$ is a unitary column vector $[1, M-1]$, and $\otimes$ is the Kronecker product.

Multiplying the three matrixes $\mathbf{Y} L, \mathbf{Y} R$, and $\Phi$ 3, we have

$$
\mathrm{YY}=\Phi 3 \circ \mathrm{Y} L^{*} \circ \mathrm{Y} R .
$$

Eventually, for each range bin, a summation over the antenna pairs is carried out: the result is then averaged over a chosen 
TABLE 1: Baselines table.

\begin{tabular}{cc}
\hline Sensor & Normal baseline \\
\hline 0 & 0 \\
1 & -470 \\
2 & -310 \\
3 & 100 \\
4 & 330 \\
5 & 580 \\
\hline
\end{tabular}

length. Moreover, an azimuth multilook (averaging) could be introduced on the result of the signal beat: $\mathrm{Y} L^{*} \circ \mathrm{Y} R$.

These operations are repeated for each testing PD excess and a maximization of the result (which is real by definition) is carried out. Finally the operation is repeated for the available range lines.

Note that within this implementation scheme the introduction of weights can be carried out by using

$$
\mathbf{Y Y}=\mathbf{Y} L^{*} \circ \Phi 3 \circ \mathbf{W} \circ \mathbf{Y} R
$$

where $\mathbf{W}$ is a weighting $3 \mathrm{D}$ matrix (symmetric for each range line), accounting for interferometric weights, see Section 4.1: it possibly varies throughout the range.

\section{SIMULATION RESULTS}

The MB-InSAR algorithm has been tested on simulated data relative to the Vesuvio area in Naples. Starting from an available high-precision DEM, we simulated six acquisitions by an ERS-like system. Table 1 shows the baselines. The interferograms corresponding to the lowest (1-4 pair, $100 \mathrm{~m}$ ) and highest (2-6 pair, $1050 \mathrm{~m}$ ) baselines are shown in Figure 5 to help the reader appreciate the amount of decorrelation caused by imaging angular diversity.

The proposed algorithm, applied with respect to the pixel-to-pixel range phase differences (PD), tackles possible constant phase offset dependence in the available interferograms, allowing a more realistic scenario. The first baseline $(-470 \mathrm{~m})$ is taken as the reference, therefore the PD on the first baseline pair are the unknowns. Figure 6 shows the PD evaluated from the noiseless, nonwrapped fringe pattern, assumed as a reference, and its histogram: note that PD associated to the flat Earth amounts to about $-1.14 \mathrm{rads} / \mathrm{pixel}$, whereas a large part of the PD is around -1.74 rads/pixel because the imaged area is located in a flank of the volcano.

The first experiment carried out was aimed at showing the reconstruction performance achievable by a single baseline measurement on the reference baseline PD. In particular we chose the lowest baseline, that is, sensor $0-3$ in Table 1. In this experiment we have also assumed no a priori information about the topography, that is, we referred to the PD of a flat Earth. As a consequence we subtracted

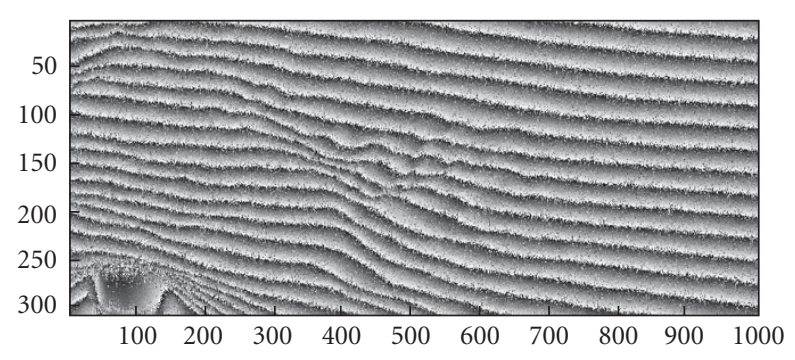

(a)

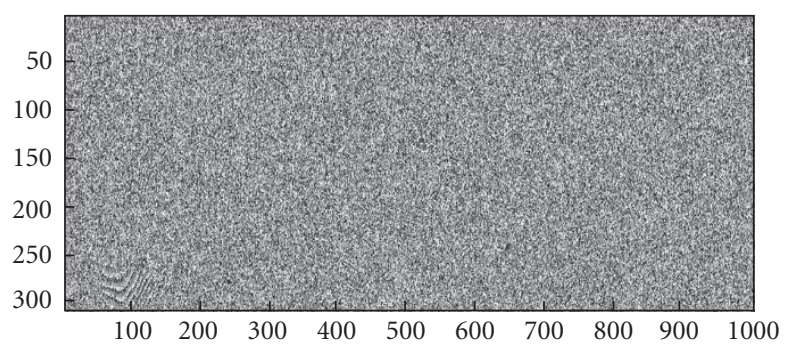

(b)

Figure 5: (a) Lowest and (b) highest baseline interferograms, 300 range pixels by 1000 azimuth pixels.

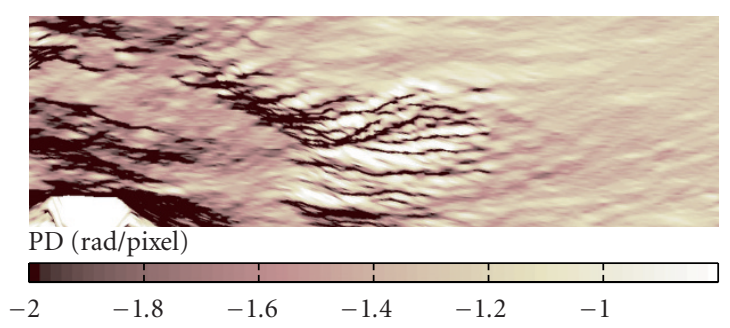

(a)

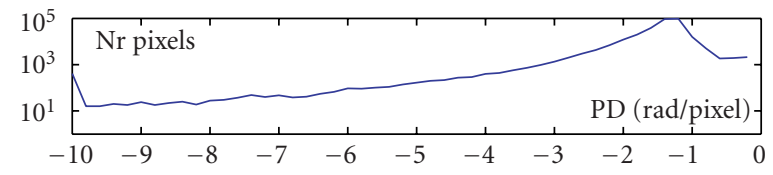

(b)

Figure 6: (a) Wanted PD image scaled in the $[-3: 0]$ interval to retain the dynamic and (b) the $\mathrm{PD}$ histogram.

from the interferograms the fringe pattern associated with flat Earth and estimated the residual PD from the flattened interferograms: the output, as for all the subsequent experiments, is the estimated PD on the reference baseline $(0-1$ in our case). Nonetheless we did not include any spectral shift filtering, that is, we let $\widehat{\boldsymbol{\Phi}}_{i j}=\mathbf{I}_{D}$ in the multiplication in (41) and $\widehat{\boldsymbol{\Phi}}_{j i}=\mathbf{I}_{D}$ in (42). The PD obtained using this interferogram pair ( $-470 \mathrm{~m}$ baseline), averaged on a window length of 5 range pixels, is shown in Figure $7 \mathrm{a}$ whereas 


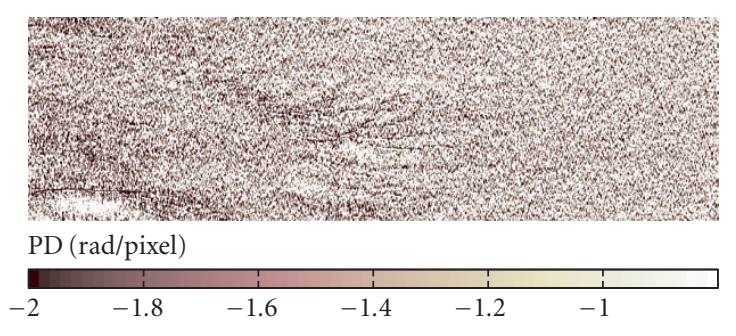

(a)

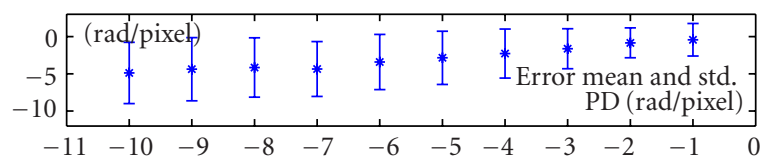

(b)

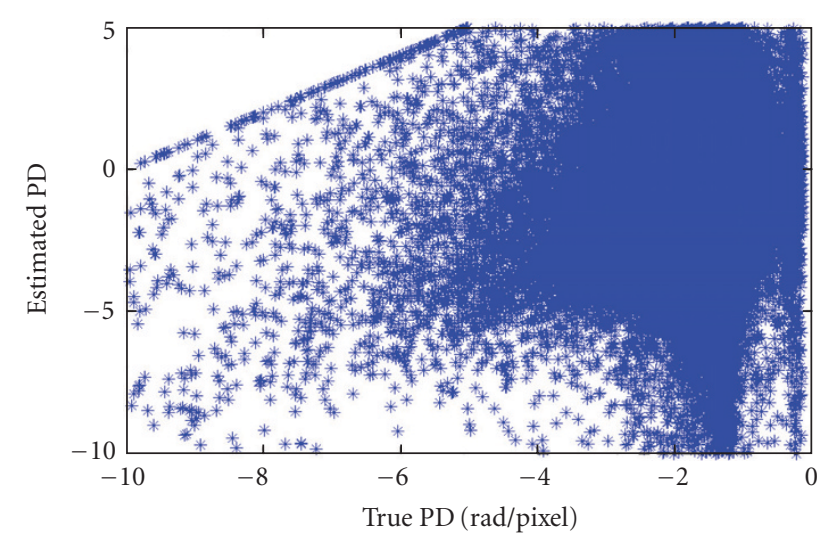

(c)

FIgURE 7: (a) PD estimated with the lowest baseline interferograms, (b) error plot (mean and standard deviation of the error), and (c) the scatter plot of the estimated PD versus the true PD.

the error bar plot (mean standard deviation of the error) is shown in Figure 7b. Here we see the poor quality of the reconstruction due to the relatively large baseline, which is confirmed by the appearance of a bias in the error plot for high slopes (ideally this should be horizontal) and by the presence of relatively high standard deviations, as well as high dispersion of the scatter plot of the estimated PD versus the true $\mathrm{PD}$ in Figure $7 \mathrm{c}$. The quality of the reconstruction improves when CB filtering was carried out: this is clearly shown in Figure 8 which presents the images of Figure 7 after CB filtering with respect to flat Earth. Nevertheless, by looking at the error plot we again recognize that it is even more tilted than before, in particular, for the steepest slopes, that is, high $\mathrm{PD}$, the estimates are strongly down-biased. The same considerations can be carried out by comparing the two scatter plots. Bias is eliminated when $\mathrm{CB}$ filtering is carried out with respect to the true PD. This is clearly evident in

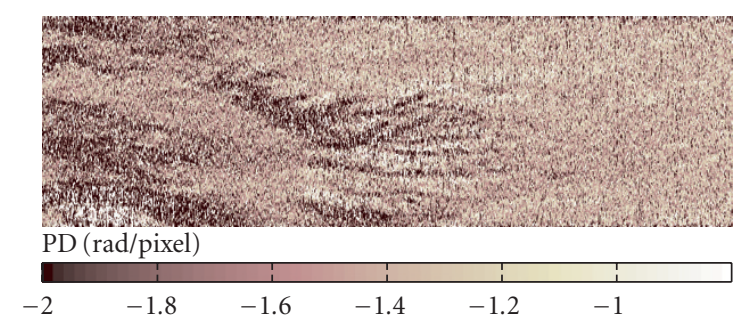

(a)

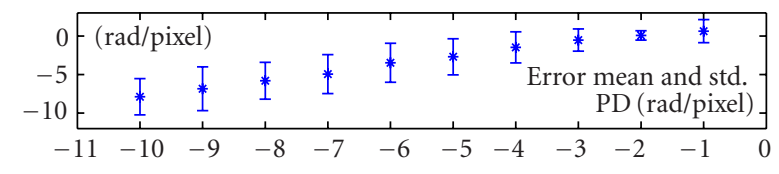

(b)

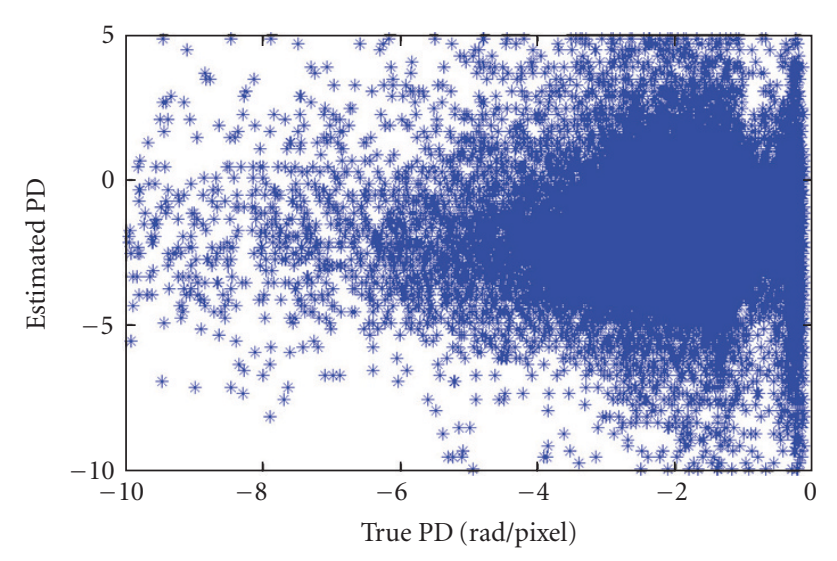

(c)

Figure 8: The same as Figure 7, but with the application of the CB with respect to the flat Earth.

Figure 9: the error bar plot is horizontal, although there is a marked dispersion. Figure 10 shows the results achieved by exploiting all the available acquisitions and the CB filtering with respect to the flat Earth. This figure, when compared Figure 8, shows that the introduction of large baseline interferograms has significantly deteriorated the estimation of high slopes (from $-3 \mathrm{rad} /$ pixel to $-2 \mathrm{rad} / \mathrm{pixel}$ ). However, it also shows the effectiveness of the CB filtering; in fact, slopes close to that of the flat Earth (in bright areas) are better reconstructed when compared to Figure 8 , see also Figure 6.

The last experiment in Figure 11 shows the best case when the $\mathrm{CB}$ filtering was tuned according to the true PD and all the acquisitions were used. Note that, usually a rather precise external DEM is always used in InSAR processing, especially when differential interferograms are produced to detect small ground movements: with this regard, DEM provided 


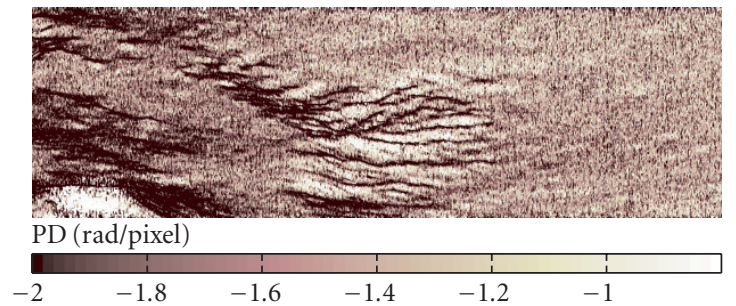

(a)

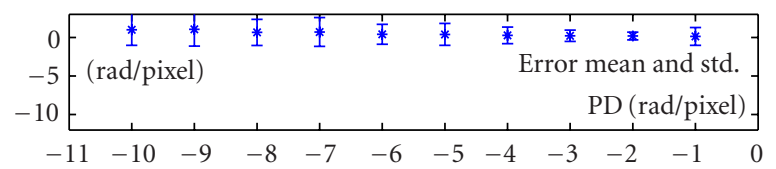

(b)

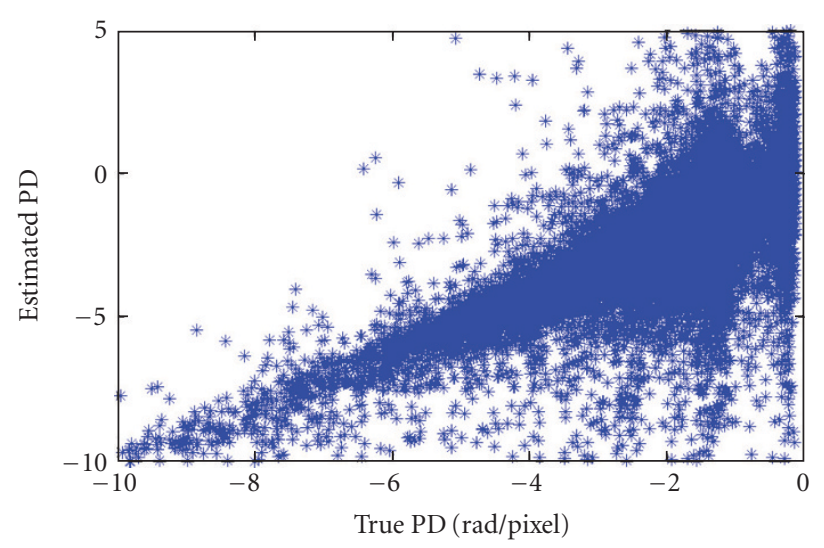

(c)

FIgURE 9: The same as Figure 8, but with the application of the CB with respect to the true DEM.

by the shuttle radar topography mission (SRTM) with $90 \mathrm{~m}$ posting and an accuracy of $15 \mathrm{~m}$ is a good reference for the estimation procedure at hands. Figure 11 shows the estimated $\mathrm{PD}$, the mean and the standard deviation, and the scatter plot. Comparing this figure with 9 and 10 and the true one, that is, Figure 6, we appreciate the improvement in the estimation of both low and high slopes: the error plot bar in the middle image is thin and horizontal whereas the scatter plot is mostly concentrated around the diagonal.

\section{CONCLUSIONS}

A general framework that links the problem of multi-baseline SAR interferometry with the single-input multiple-output multichannel estimate has been established. We have shown that one of the most popular techniques to approach the problem, namely cross-relations, can be extended to the MB-InSAR case with slight modifications. The LS solution of

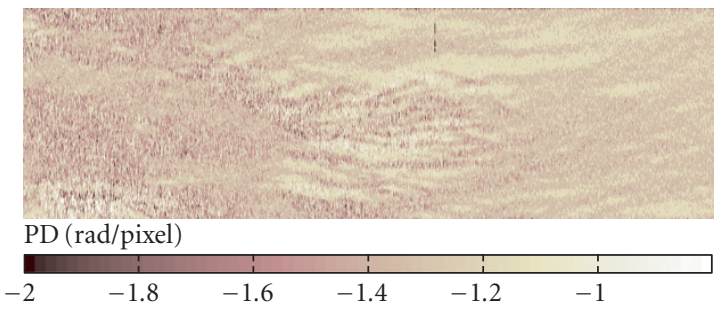

(a)

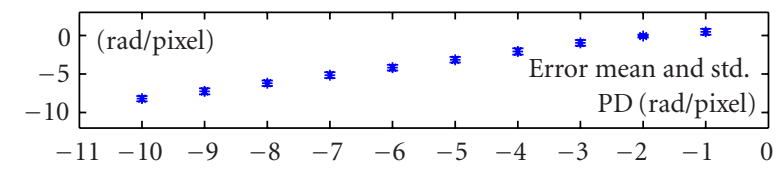

(b)

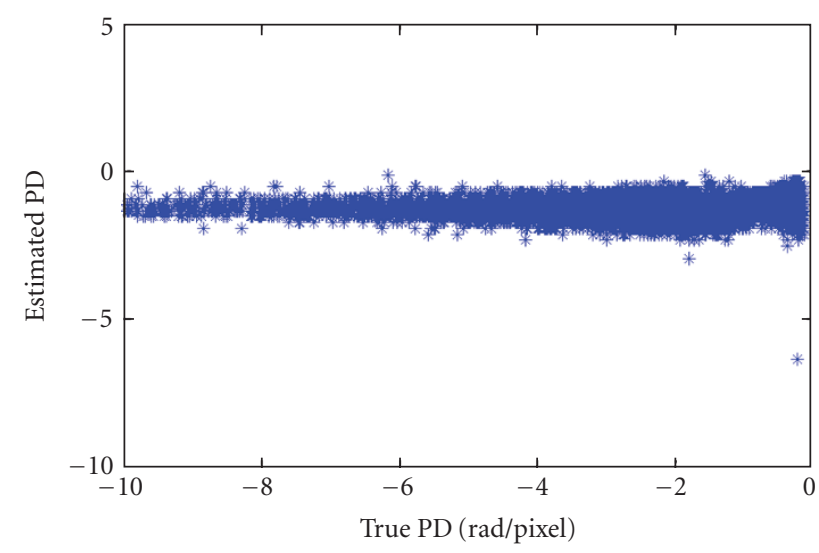

(c)

Figure 10: The same as Figure 8, but with using all the interferometric pairs.

the thus derived equation system leads to the maximization of the total energy that comes from taking all the possible interferograms. Not surprisingly, the outcome of this technique is that in forming each interferogram the two image pairs are prefiltered by a common band filter; such filtering corresponds to the suboptimal spectral-shift approach already known in literature. An efficient implementation has been shown for the estimate of a constant terrain slope. The simulation of MB ERS-like acquisitions in rough topography has led to interesting results that reel the potential of the technique.

\section{ACKNOWLEDGMENTS}

This work has been committed under ASI Contract I/R/ 169/02 Multiple pass SAR Interferometry for very accurate Earth modeling (2003-2004). The authors wish to thank the Italian Space Agency (ASI) for sponsoring the work. 


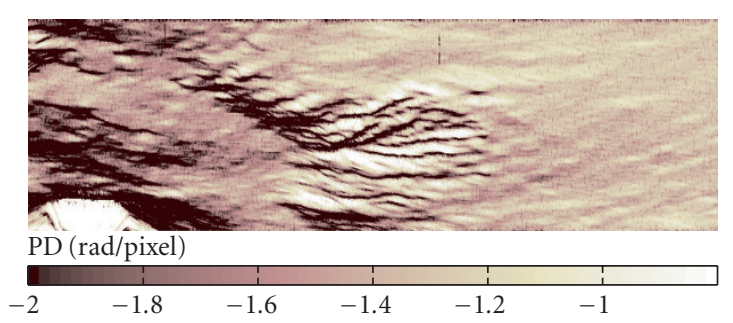

(a)

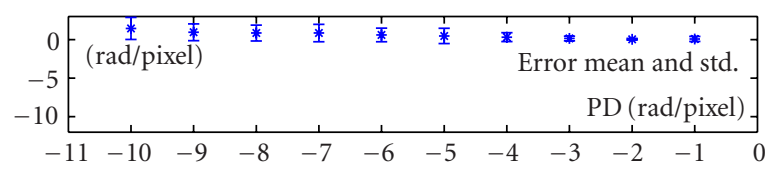

(b)

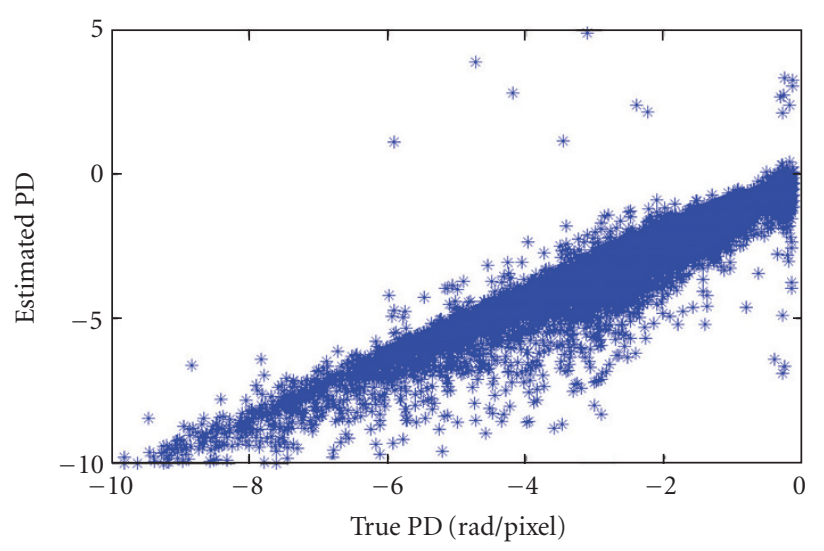

(c)

FIgURE 11: (a) PD estimated with all interferograms pairs and the application of the CB filtering with respect to the true topography. (b) The associated error plot (mean and standard deviation of the error). (c) The scatter plot of the estimated PD versus the true PD.

\section{REFERENCES}

[1] A. Ferretti, C. Prati, and F. Rocca, "Permanent scatterers in SAR interferometry," IEEE Trans. Geosci. Remote Sensing, vol. 39, no. 1, pp. 8-20, 2001.

[2] F. Gatelli, A. Monti Guarnieri, F. Parizzi, P. Pasquali, C. Prati, and F. Rocca, "The wavenumber shift in SAR interferometry," IEEE Trans. Geosci. Remote Sensing, vol. 32, no. 4, pp. 855-865, 1994.

[3] G. W. Davidson and R. Bamler, "Multiresolution phase unwrapping for SAR interferometry," IEEE Trans. Geosci. Remote Sensing, vol. 37, no. 1, pp. 163-174, 1999.

[4] A. Monti Guarnieri and F. Rocca, "Combination of low- and high-resolution SAR images for differential interferometry," IEEE Trans. Geosci. Remote Sensing, vol. 37, no. 4, pp. 20352049, 1999.

[5] G. Fornaro and A. Monti Guarnieri, "Minimum mean square error space-varying filtering of interferometric SAR data," IEEE Trans. Geosci. Remote Sensing, vol. 40, no. 1, pp. 11-21, 2002.
[6] A. Ferretti, A. Monti Guarnieri, C. Prati, and F. Rocca, "Multi baseline interferometric techniques and applications," in Proc. ESA Workshop on Applications of ERS SAR Interferometry (FRINGE '96), Zürich, Switzerland, September-October 1996.

[7] R. Bamler and P. Hartl, "Synthetic aperture radar interferometry," Inverse Problems, vol. 14, no. 4, pp. R1-R54, 1998.

[8] P. A. Rosen, S. Hensley, I. R. Joughin, et al., "Synthetic aperture radar interferometry," Proc. IEEE, vol. 88, no. 3, pp. 333382, 2000.

[9] D. C. Ghiglia and M. D. Pritt, Two-Dimensional Phase Unwrapping: Theory, Algorithms, and Software, John Wiley \& Sons, New York, NY, USA, 1998.

[10] G. Franceschetti and G. Fornaro, "Synthetic aperture radar interferometry," in Synthetic Aperture Radar Processing, G. Franceschetti and R. Lanari, Eds., chapter 4, pp. 167-223, CRC Press, Boca Raton, Fla, USA, 1999.

[11] H. A. Zebker and J. Villasenor, "Decorrelation in interferometric radar echoes," IEEE Trans. Geosci. Remote Sensing, vol. 30, no. 5, pp. 950-959, 1992.

[12] L. Tong and S. Perreau, "Multichannel blind identification: from subspace to maximum likelihood methods," Proc. IEEE, vol. 86, no. 10, pp. 1951-1968, 1998.

[13] G. Xu, H. Liu, L. Tong, and T. Kailath, "A least-squares approach to blind channel identification," IEEE Trans. Signal Processing, vol. 43, no. 12, pp. 2982-2993, 1995.

G. Fornaro received the Laurea degree summa cum laude in electronic engineering in 1992 and the Ph.D. degree in 1997 from the University "Federico II," Napoli, Italy. Since 1996, he is with the "Institute for Electromagnetic Sensing of the Environment" (IREA) of the Italian National Research Council (CNR), where he currently holds the position of Senior Researcher. In the past, he has been an Adjunct Professor

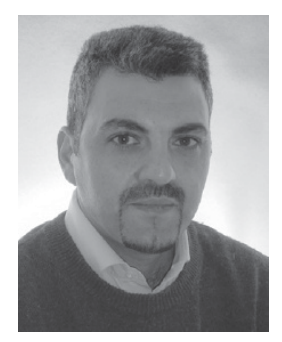
of signal theory and communication in several Italian universities: Napoli Federico II, Cassino, and Reggio Calabria. His main research interests are in the signal processing filed with applications to synthetic aperture radar (SAR) data processing from airborne and spaceborne systems, including motion compensation, multichannel SAR interferometry, differential SAR interferometry, and 3D SAR focusing. Dr. Fornaro has been a Visiting Scientist at the German Aerospace Establishment (DLR) and at the Politecnico of Milano and he has been a Lecturer in several universities and international institutions such as the Istituto Tecnológico de Aeronáutica (ITA) in Sao José dos Campos (Brazil) and Remote Sensing Technology Center (RESTEC), Tokyo. He is currently responsible for the Remote Sensing Unit of the Regional Center of Competence "Analysis and Monitoring of the Environmental Risk" funded by the European Community on Provision 3.16. Dr. Fornaro was awarded (1997) the Mountbatten Premium by the Institution of Electrical Engineers (IEE).

A. Monti Guarnieri was born in Milano, Italy, on February 9, 1962. He received the "Laurea" (cum laude) degree in electronic engineering from Politecnico di Milano in 1988, and since that date, he has worked in the Electronic Engineering Department where he joined the Digital Signal Processing Team. He is currently an Associate Professor in the same university.

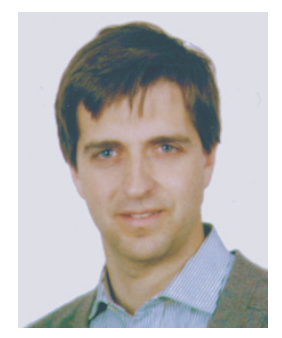


Present and past courses included "signals and systems," "signal theory," "digital signal processing," "algorithms and circuits for telecommunications," and "radar theory and technique." His research interests concern digital signal processing, mainly in the field of synthetic aperture radar signal processing. Since 1987, he has authored/coauthored about 100 scientific publications in the field of synthetic aperture radar. Professor Monti Guarnieri was awarded the "Symposium Paper Award" at the IGARSS' 89 and the "Best Paper Award" at EUSAR 2004.

A. Pauciullo was born in Cercola, Italy, on October 10, 1969. He received the Dr. Eng. degree with honors in 1998 and the Ph.D. degree in information engineering in 2003, both from the University of Naples, Italy. Since 2001, he has been with the "Institute for Electromagnetic Sensing of the Environment" (IREA) of the Italian National Research Council (CNR), where he holds a position of Researcher, and since 2003, he has

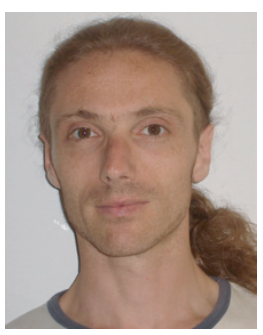
been an Adjunct Professor of signal theory at the University of Cassino (Italy). His current research interests regard the field of statistical signal processing with emphasis on synthetic aperture radar processing and CDMA systems.

S. Tebaldini was born in Milan, Italy on July 1, 1974. He received the Diploma degree (cum laude) in telecommunication engineering in 2000, with a thesis on digital PLL, and the Laurea degree (cum laude) in 2005, with a thesis on surface topography reconstruction by means of multi-baseline SAR interferometry, both from Politecnico di Milano. In 2003, he joined the "GSA" Project, working on an innovatory time/ frequency distribution system to be developed for ESA. 